\title{
Immigrants Are Like... The Representation of Immigrants in Italy: The Metaphors Used by Students and Their Family Backgrounds
}

\section{Elisa Cavicchiolo - Fabio Alivernini - Sara Manganelli}

Istituto Nazionale per la Valutazione del Sistema Educativo di Istruzione e Formazione (INVALSI) (Italy)

doi: 10.7358/ecps-2016-013-cavi fabio.alivernini@gmail.com elisa.cavicchiolo@gmail.com sara.manganelli@gmail.com

\author{
GLI IMMIGRATI SONO COME ... LA RAPPRESENTAZIONE \\ DEGLI IMMIGRATI IN ITALIA: LE METAFORE DEGLI STUDENTI \\ E IL BACKGROUND FAMILIARE DI PROVENIENZA
}

\section{Abstract}

The aim of this study is to identify the way young Italian students represent immigrants and to evaluate the existence of relationships between these representations and the family background of the students. The data is based on a sample of Italian students $(N=841)$. The respondents were given an open question, which asked them to provide a simile or a metaphor concerning immigrants. A mixed methodology was employed, which involved: (i) analysis of the qualitative content; (ii) quantitative analysis investigating the relationship between the categories identified by means of an analysis of the content and the socioeconomic status of the student. The results showed a very diverse and articulated set of representations, with the contents of the metaphors produced by the students ranging from those based on a kind of «us-them» opposition, to images based on an attitude of tolerance and equality, and semantic regions with a more emotional component. The analysis revealed some statistically significant relationships between the socio-economic backgrounds of the students' families and some types of metaphors produced by the students. The mixed research 
methodology adopted proved to be effective with respect to the objectives of the study, and it combines the use of reliable statistical tools with the qualitative richness of metaphor-based answers to open questions.

Keywords: Immigrants, Mental representations, Metaphors, Mixed-method approach, Socioeconomic status.

\section{INTRODUZIONE}

La scuola è una delle strutture sociali fondanti nello sviluppo degli individui e luogo naturale di apprendimento inteso in senso ampio, non solo come acquisizione di conoscenze, ma anche come sviluppo di valori, atteggiamenti e comportamenti. Negli ultimi anni, un tema che è entrato con forza nel dibattito sulla scuola è l'immigrazione. Come è noto, il peso del fenomeno migratorio ha avuto un aumento considerevole negli ultimi venticinque anni in Italia e questo nel mondo scolastico si è tradotto in una riflessione sulla composizione delle classi, che tendono ad essere sempre più eterogenee e nella introduzione di policy e strategie didattiche a favore di integrazione, cooperazione e partecipazione. Il sistema scolastico italiano vede un aumento ormai sistemico e non più oscillatorio della popolazione straniera che è passata da 196.414 alunni nell'anno scolastico 2001/2002 a 629.360 dell'anno scolastico 2008/2009 (anno di riferimento dei dati di questo studio) fino agli 802.844 dell'anno scolastico 2013/2014, con una forte presenza di stranieri nati in Italia (233.033 nell'anno scolastico 2008/2009 e 415.283 nell'anno scolastico 2013/2014).

Essere studenti stranieri in Italia oggi significa avere una maggiore probabilità di ritardo scolastico nei vari ordini di scuola e una minore probabilità di essere ammessi ai gradi superiori d'istruzione (MIUR-ISMU, 2015). I dati INVALSI (Istituto Nazionale per la Valutazione del Sistema Educativo di Istruzione e di Formazione) mostrano come gli alunni stranieri ottengano risultati sistematicamente più bassi dei loro compagni in matematica e soprattutto in italiano, con una forbice che si riduce nel caso delle seconde generazioni (INVALSI, 2015). Le indagini internazionali sono in linea con i dati rilevati a livello nazionale. L'indagine OCSE PISA (Programme for International Student Assessment) evidenzia come gli studenti immigrati abbiano punteggi inferiori in matematica (punteggio medio di 442 rispetto al 490 degli Italiani) e nelle competenze di lettura (punteggio medio di 432 rispetto ai coetanei italiani di 497), con un quadro più eterogeneo in parti- 
colare per le seconde generazioni (INVALSI, 2012a). Anche i risultati delle indagini internazionali IEA (International Association for the Evaluation of Educational Achievement), PIRLS (Progress in International Reading Literacy Study) e TIMMS (Trends in International Mathematics and Science Study) mostrano come i punteggi medi in lettura da un lato e in matematica dall'altro siano più bassi per gli alunni stranieri (INVALSI, 2012b). Inoltre dati recenti a livello nazionale (Alivernini \& Manganelli, 2016) hanno messo in luce come essere studenti stranieri in Italia significhi essere socialmente più isolati in classe, con possibili conseguenze per il benessere psicologico a scuola di questi alunni. La scuola si presenta quindi come un ambiente di studio in cui il fenomeno migratorio e le sue evoluzioni sono particolarmente evidenti: si caratterizza per un contatto e una convivenza quotidiana tra studenti, omogenei per classe d'età, ma spesso non per paese e cultura di provenienza. La classe è un ambiente in cui si formano gruppi, amicizie, che possono prescindere o meno dall'appartenenza geografica. Alcuni studi a scuola hanno per esempio evidenziato come sia difficile mantenere amicizie intergruppo e come questi rapporti dipendano dalle pressioni sociali del proprio gruppo di appartenenza e dal desiderio di essere accettati (Castelli et al., 2007). A scuola dunque, rispetto all'immigrazione, si definiscono e ridefiniscono valori, atteggiamenti e percezioni tra studenti sia a livello individuale, che collettivo.

\subsection{Le rappresentazioni dell'immigrato: teorie e ricerche empiriche}

Secondo Moscovici (1984), le rappresentazioni sono veri e propri sistemi cognitivi, che possiedono un loro specifico linguaggio e che consentono alle persone di classificare oggetti e persone (Moscovici, 1988). La letteratura sulle rappresentazioni mentali verso gli immigrati è molto variegata e attinge a diversi campi di ricerca psicologica e sociale. Spesso gli studi teorici ed empirici si sono concentrati sull'individuazione di determinanti che possono avere una relazione con atteggiamenti positivi o negativi rispetto al gruppo di immigrati. Ad esempio le teorie del contatto (Intergroup Contact Theory) hanno individuato nel contatto interpersonale un moderatore degli atteggiamenti negativi rispetto al gruppo di non appartenenza (Allport, 1954; Pettigrew, 1998; Pettigrew \& Tropp, 2006). Secondo queste teorie, le occasioni di entrare in relazioni di tipo cooperativo e positivo avrebbero quindi un effetto sulla riduzione del pregiudizio e delle situazioni di conflitto. Allport (1954) ha individuato alcune caratteristiche facilitanti per assicurare un contatto efficace tra i gruppi: la condivisione di obiettivi e la cooperazione, l'interazione informale con l'altro, la presenza di un'autorità a supporto del contatto, 
l'appartenenza allo stesso status sociale. La scuola è stato uno dei campi di osservazione privilegiato proprio per le sue caratteristiche strutturali: la scuola è uno dei luoghi in cui il contatto interculturale tra studenti e tra studenti e docenti avviene quotidianamente. Le ricerche empiriche hanno permesso di indagare il rapporto tra le relazioni interpersonali e i pregiudizi e gli stereotipi contribuendo all'individuazione di variabili in grado di favorire, seppur debolmente, una serie di atteggiamenti positivi rispetto agli studenti immigrati. In questo senso, diversi studi hanno messo in evidenza come il dialogo in classe, il clima di classe e la discussione su temi civici possano avere un effetto positivo sugli atteggiamenti verso gli immigrati (Barber, Purta, \& Fennelly, 2010; Caro \& Schulz, 2012; Isac, Maslowski, \& van der Werf, 2012).

Le rappresentazioni e gli atteggiamenti verso l'altro possono essere però anche di natura conflittuale. Un altro filone di teorie si è concentrato proprio sul conflitto e sulla formazione di atteggiamenti intergruppo negativi. Secondo queste teorie (Sherif, 1966; Sherif \& Sherif, 1969) un determinato gruppo ne percepisce un altro (a cui non appartiene) come una minaccia per la realizzazione dei propri interessi. La minaccia può essere di tipo reale o percepito. Secondo la Realistic Conflict Theory (Coser, 1956; Blumer, 1958; Blalock, 1967), la competizione tra i gruppi avviene per interessi per lo più reali, per risorse di tipo economico o politico e può dare origine ad atteggiamenti di tipo discriminatorio. Per la Intergroup Threat Theory (Stephan, Ybarra, \& Bachman, 1999) la tipologia di minaccia che caratterizza la relazione intergruppo è legata principalmente alla percezione delle condizioni di competizione più che alla natura reale dell'interesse. Secondo questo approccio, le minacce possono essere anche di tipo simbolico, legate a valori, norme e simboli e possono influenzare l'atteggiamento verso il gruppo di non appartenenza. Infine un altro esempio d'ipotesi teorica è la Social Identity Theory, sviluppata da Tajfel e colleghi (Tajfel \& Turner, 1979; Tajfel, 1982). Questa teoria ha analizzato i processi di categorizzazione e di identificazione sociale che sono alla base dello sviluppo di specifici processi valutativi volti a favore del proprio gruppo di appartenenza (in-group) rispetto all'altro (outgroup).

Le ricerche di tipo empirico sulle rappresentazioni mentali e i pregiudizi si sono concentrate prevalentemente sullo studio degli atteggiamenti verso l'altro e sull'analisi dei processi che portano alla costruzione di queste immagini. L'immagine che gli studenti hanno degli immigrati è stata ad oggi studiata perlopiù in termini di atteggiamenti, vale a dire sulla base di valutazioni più o meno positive di un gruppo di persone o di aspetti che li riguardano (Bohner \& Dickel, 2011). In Italia, un esempio su larga scala di questo tipo di ricerche è costituito dall'indagine IEA su Educazione Civica e Cittadinanza (IEA ICCS - International Civic and Citizenship Education Study), la 
cui ultima edizione conclusa risale al 2009. L'indagine ICCS 2009 si poneva l'obiettivo più generale di raccogliere evidenze empiriche su aspetti cognitivi e atteggiamenti relativi a temi di educazione civica e cittadinanza. Lindagine era rivolta a un campione rappresentativo di studenti all'ottavo grado di istruzione (in Italia il campione ha riguardato gli studenti all'ultimo anno di scuola secondaria di primo grado) e prevedeva la partecipazione di trentotto paesi, tra cui appunto l'Italia. Rispetto all'ambito delle convinzioni e degli atteggiamenti degli studenti, il questionario promosso da IEA raccoglieva, tra le altre, informazioni rispetto all'uguaglianza di diritti e nello specifico indagava gli atteggiamenti degli studenti verso i diritti degli immigrati. I punteggi medi ottenuti dall'Italia rispetto a questa dimensione hanno mostrato un atteggiamento meno favorevole degli studenti italiani (in particolar modo i maschi) rispetto alla media internazionale (l'Italia si è caratterizzata per un punteggio medio pari a 48,4 rispetto alla media ICCS di 50). Un altro dato che è emerso dalle analisi della IEA è stato l'associazione tra lo status socioeconomico degli studenti (SES) e i loro atteggiamenti rispetto agli immigrati. I risultati hanno messo in luce come a livelli più elevati di SES corrispondano atteggiamenti più positivi rispetto ai diritti degli immigrati (Barber, Torney-Purta, \& Fennelly, 2010; Isac, Maslowski, \& van der Werf, 2012). Una possibile interpretazione (Caro, \& Schulz, 2012) di questa relazione è che gli studenti con un livello più basso di background socioeconomico percepiscano gli immigrati come una eventuale minaccia ai propri interessi, in competizione per le stesse risorse. Lo status socioeconomico può avere un'influenza nelle rappresentazioni della società e nella distanza sociale tra i gruppi (Mosso, 2007).

Le misure degli atteggiamenti come quelle appena presentate hanno ricevuto critiche in letteratura in quanto risentono molto della desiderabilità sociale (Krosnick, Judd, \& Wittenbrink, 2005). Un ulteriore limite di queste misure riguarda le problematiche associate all'utilizzo di strumenti quantitativi basati su domande chiuse. Tali strumenti sono infatti limitati nella loro possibilità di scoprire aspetti non conosciuti di un fenomeno, in quanto misurano costrutti concettualizzati a priori dal ricercatore, che vengono definiti operativamente e trasformati in variabili prima di raccogliere i dati (Lucidi, Alivernini, \& Pedon, 2008; Alivernini, 2012; Alivernini et al., 2015).

L'obiettivo generale di questo studio è stato quello di esplorare le rappresentazioni che gli studenti hanno degli immigrati attraverso lo strumento delle domande aperte basate su similitudini e metafore. Lo stimolo aperto ha offerto il vantaggio di poter cogliere aspetti più impliciti degli atteggiamenti degli studenti e ha consentito un'esplorazione delle percezioni degli studenti in questo ambito non vincolata a priori. La domanda aperta è più vicina all'esperienza dello studente e permette di indagare competenze più complesse 
(Intraversato \& Lucisano, 2013). La metodologia adottata è stata di ricerca mista: dopo aver utilizzato strumenti aperti e un analisi del contenuto qualitativa, è stato utilizzato un approccio quantitativo per valutare attraverso test statistici, le relazioni emerse tra le immagini degli immigrati e il background socioeconomico degli studenti.

Sebbene lo studio sia di tipo esplorativo, è stato possibile formulare alcune ipotesi generali che hanno guidato le diverse fasi di ricerca. In particolare:

- La prima ipotesi è che le rappresentazioni sugli immigrati siano più ricche e più articolate rispetto a quelle fornite dall'insieme di teorie e delle ricerche empiriche fin qui considerate. La letteratura, in particolare a scuola, si è concentrata per lo più sull'individuazione delle determinanti degli atteggiamenti, piuttosto che analizzare nel dettaglio i contenuti degli atteggiamenti stessi. I dati empirici hanno raccolto informazioni per lo più di tipo valutativo, basate essenzialmente sulle dicotomie buono/cattivo, positivo/negativo, non restituendo appieno la forza e la varietà delle immagini dell'immigrato. Attraverso questo studio ci si aspetterebbe di trovare un numero di dimensioni semantiche maggiori rispetto a quelle che caratterizzano gli studi sugli stereotipi, tenendo conto della complessità delle dimensioni di contenuto e di processo (Fiske et al., 2002; Lee \& Fiske, 2006).

- La seconda ipotesi è che le rappresentazioni sugli immigrati abbiano una relazione con lo status socioeconomico (SES) dello studente. Il SES è infatti una variabile di cui molti studi hanno mostrato avere una relazione con gli atteggiamenti verso gli immigrati misurati in modo tradizionale (Lee \& Fiske, 2006).

\subsection{La similitudine come strumento per lo studio delle rappresentazioni mentali}

Le caratteristiche degli stimoli e degli strumenti di misura proposti giocano un ruolo centrale per salvaguardare la ricchezza e il punto di vista dei soggetti coinvolti, in particolare in presenza di tematiche che implicano un giudizio, un valore o che sono riconducibili a domini sensibili a livello individuale o collettivo. Le similitudini e più in generale il ricondursi a dimensioni figurate ed esemplificative possono essere d'aiuto per lo studio di concetti complessi (Vosniadou \& Ortony, 1989). Uno dei passaggi chiave nell'utilizzo della metafora come strumento di studio è costituito dai lavori di Lakoff e Johnson che a partire dalla fine degli anni '70 contribuiscono ad inserire la metafora nell'ambito della psicologia cognitiva (Lakoff \& Johnson, 1980). La metafo-

\footnotetext{
ECPS Journal - 13/2016

http://www.ledonline.it/ECPS-Journal/
} 
ra dà forma al pensiero e consente di descrivere e capire la realtà; in questa visione il modo in cui si pensa ed agisce ha una natura metaforica (Lakoff \& Johnson, 1980). La similitudine quindi comincia ad essere concettualizzata non solo come una figura del discorso, ma in termini di rappresentazione mentale (Scalera, Palmerio, \& Alivernini, 2007), come un processo inevitabile del pensiero umano (Kövecses, 2010). Secondo questo approccio cognitivista, la metafora consente la rappresentazione (e la comprensione) di un dominio concettuale detto target (nel caso di questo studio gli immigrati) in termini di un altro dominio concettuale detto source. La similitudine colma dei vuoti lessicali e consente di ricostruire la realtà, ma gioca anche un'opera selettiva, di scelta di una particolare immagine (e quindi di un modello mentale) piuttosto che un altro (Scalera et al., 2007). Nell'articolo citato, Scalera e colleghi riportano una metafora molto utilizzata per l'interpretazione dei fenomeni educativi, l'educazione come cura. Si parla quindi dell'educazione come «una terapia» e di un mondo scolastico popolato da "pazienti» (gli studenti), «medici» (gli insegnanti), da «sistemi scolastici in buono stato di salute», da «situazioni di emergenza». Questo esempio mostra come la concettualizzazione di «educazione» passi attraverso immagini appartenenti ad un altro dominio mentale e rappresenti (e plasmi) il modo con cui si pensa e agisce (Lakoff \& Johnson, 1980). Il costrutto metaforico crea delle corrispondenze inconsuete ("uno studente a rischio», "una scuola che soffre»), ma che sono concettualmente interessanti e che consentono di dare un'immagine viva e riconoscibile di un dominio complesso. In questi approcci la natura della metafora e del paragone è riconducibile ai modelli mentali (Johnson-Laird, 1989; Lakoff, 1993) e si configura quindi come uno strumento potenzialmente molto utile per l'identificazione delle rappresentazioni che gli studenti hanno degli immigrati.

Il presente lavoro utilizza questo strumento per esplorare le immagini degli studenti rispetto agli immigrati in Italia e la relazione tra queste immagini e il background socioeconomico di appartenenza.

\section{Obiettivi}

Gli obiettivi dello studio sono:

- individuare i concetti chiave che sono alla base delle rappresentazioni degli studenti rispetto gli immigrati, tenendo conto della loro complessità e ricchezza;

- analizzare la relazione tra i concetti emersi e il background socioeconomico degli studenti. 


\section{Metodo}

\subsection{Il campione}

La popolazione presa in esame per questo studio è costituita da studenti della scuola italiana $(\mathrm{N}=841)$ all'ottavo grado di istruzione (con un età media di 13,5 anni). Gli studenti sono un campione casuale estratto da un gruppo più ampio di giovani $(\mathrm{N}=3.366)$ coinvolto nell'indagine IEA ICCS 2009 su educazione civica e cittadinanza. Il metodo di campionamento è stato di tipo casuale e la procedura di selezione è stata eseguita attraverso il programma SPSS utilizzando all'interno comando «Select cases» l'opzione "Random sample of cases» e impostando la percentuale approssimativa di casi da prendere in considerazione (circa il $25 \%$ del totale del campione). Il campione oggetto di questo studio costituisce un quarto del totale degli studenti partecipanti allo studio in Italia.

La composizione degli studenti all'interno delle classi vede la presenza percentuale di circa il 54\% di maschi e di circa il $46 \%$ di femmine, mentre la percentuale di studenti stranieri si assesta intorno al 9\%. Rispetto alle macroaree territoriali di appartenenza, gli studenti di questo sottogruppo provengono per circa il $13 \%$ dal Nord-Est, per il $23 \%$ dal Nord-Ovest, per il $20 \%$ dal Centro, per il 27\% dal Sud e per il 17\% circa dal Sud e Isole. Il SES presenta una media pari a -0,06 e una deviazione standard pari 1,01 e non si discosta quindi dalla distribuzione del SES nel campione più generale di studenti che hanno partecipato all'indagine ICCS in Italia.

L'indagine è stata condotta nel 2009 ed è attualmente la fonte di dati più recente disponibile.

La scelta di lavorare con una parte del campione totale di studenti è stata dettata dalla limitatezza delle risorse a disposizione: l'analisi qualitativa effettuata è stata molto onerosa in termini di tempo necessario per la codifica e di numero di ricercatori da coinvolgere per i controlli di attendibilità.

\subsection{Gli strumenti e le variabili}

All'interno di un questionario strutturato volto a misurare aspetti cognitivi, atteggiamenti e comportamenti, è stato chiesto agli studenti di completare un paragone avente come dominio target gli immigrati. In particolare, la domanda aperta posta agli alunni è stata: "Gli immigrati sono come ...». Gli studenti sono stati lasciati completamente liberi di rispondere, senza vincolare in alcun modo le risposte. Per quanto riguarda i dati quantitativi, è stato preso in considerazione il SES dello studente. 
Nello studio in oggetto il SES è una variabile composita, basata su tre indici: lo status occupazionale più alto dei genitori, il livello più alto di educazione dei genitori (calcolato come numero totale di anni di istruzione secondo la classificazione ISCED - International Standard Classification of Education) e il numero di libri posseduti a casa. Le tre variabili si basano sulle risposte fornite dagli studenti rispetto alla propria situazione familiare. L'indice sullo status occupazione più alto dei genitori è ottenuto attraverso una domanda aperta sul tipo di lavoro della madre e/o del padre dello studente. Le risposte sono quindi codificate rispetto ai codici ISCO (International Standard Classification of Occupations) e consentono la costruzione di diversi indici, tra cui appunto lo status occupazionale più alto dei genitori. La variabile relativa al livello più alto di educazione dei genitori è calcolata partendo dagli indici relativi al livello di educazione del padre e/o della madre dello studente categorizzati in cinque livelli ISCED. Infine l'indice sulle risorse letterarie possedute a casa è ottenuto attraverso una domanda in cui viene chiesto agli studenti di indicare il numero di libri presenti in famiglia scegliendo tra sei diverse categorie, che vanno da un minimo di 0 ad un massimo di 500 e più libri.

Il SES è ricavato attraverso una analisi in componenti principali dei tre indici descritti ed è misurato su una scala standardizzata a livello internazionale con media 0 e deviazione standard pari a 1 (Schulz, Ainley, \& Fraillon, 2011).

\subsection{Le analisi}

I dati sono stati analizzati con metodologie di analisi del contenuto (Krippendorff, 1983; Weber, 1990), con un approccio di tipo induttivo e qualitativo (Mayring, 2000; Lucidi et al., 2008). Successivamente sono state utilizzate tecniche quantitative per valutare attraverso l'uso di strumenti statistici la relazione tra le variabili in esame (Lucisano \& Salerni, 2003), nello specifico tra le caratteristiche risultanti dal processo di codifica e il background socioeconomico degli studenti. La metodologia della ricerca è stata quindi mista dove ad una prima fase di analisi di tipo descrittivo, volta a dare ricchezza e organicità ai contenuti, è seguita una fase a livello correlazionale (La Marca, 2014).

\subsubsection{Analisi del contenuto qualitativa}

Il processo di codifica ha previsto i seguenti passaggi, già utilizzati in altri studi (e.g., Alivernini et al., 2015; Brandini, 2015; Cavicchiolo, Alivernini, \& Manganelli, 2015):

- l'individuazione del contenuto centrale della metafora e del testo (eventuale) che specificava il contenuto centrale; 
- l'identificazione delle caratteristiche letterali e/o figurate relative al contenuto centrale e del testo della metafora, prendendo in considerazione solo quelle dotate di senso nell'incrocio tra i due domini (dominio immigrati e dominio metafora);

- l'attribuzione della metafora (contenuto centrale più testo) ad una o più categorie.

Il software utilizzato per le operazioni di codifica è stato $Q D A$ Miner versione 4.1.

In questo studio il dominio target è rappresentato dal concetto astratto di «immigrati» mentre le metafore costruire dagli studenti sono state le diverse source (Tabella 1).

Tabella 1. - La struttura della metafora: dominio target e dominio source, alcuni esempi.

\begin{tabular}{ll}
\hline \multicolumn{1}{c}{ Dominio TARgeT } & \multicolumn{1}{c}{ Dominio sourCE } \\
\hline Gli immigrati sono come $\ldots .$. & Dei bambini in cerca d'aiuto \\
& Fratelli \\
& Opportunisti \\
& Il pane \\
& Persone normali \\
& Delle macchine che viaggiano \\
& Delle novità \\
& Una macchia indelebile sulla camicia \\
& Le api \\
& Uccelli senza nido \\
\hline
\end{tabular}

Ad esempio, riprendendo la metafora "gli immigrati sono come dei bambini in cerca d'aiuto", i bambini costituiscono il contenuto centrale della metafora mentre «in cerca d'aiuto" è un testo che consente di ampliare il range di caratteristiche letterali/figurate riferibili al paragone. La parola «bambini» richiama una sfera concettuale riconducibile all'essere indifesi, giovani; mentre «in cerca d'aiuto" fa riferimento a persone che necessitano di un aiuto e di una protezione.

Le categorie individuate sono nate da un processo ricorsivo e bottom-up che ha previsto la creazione di una nuova categoria ogni qual volta si è presentato un tema distinto senza tener conto, nella prima fase, di un criterio di parsimonia. Il principio di attribuzione della metafora alla categoria è stato di tipo semantico, lungo una stessa scala di astrazione (Lucidi et al., 2008). La fase esplorativa ha previsto che il codificatore potesse ritornare sulle categorie create, modificandole, salendo o scendendo nel grado di astrazione, accorpandole, fino alla creazione di alcune regole stabili e generalizzabili di analisi. 
In questo modo le categorie hanno permesso di cogliere i concetti sottesi dalle similitudini, senza evidenti sovrapposizioni o ridondanze.

Una volta definito il codebook è stata fatta una valutazione formativa della sua attendibilità (Lucidi et al., 2008). Seguendo la procedura suggerita da Neuendorf (2002), il codebook è stato illustrato e discusso tra più codificatori. È stato poi selezionato un campione di metafore, codificate in modo indipendente dai diversi codificatori. Dopo aver valutato i risultati di questo processo, il codebook è stato modificato e successivamente codificato un nuovo campione di metafore. È stato quindi calcolato l'alpha ( $\alpha)$ di Krippendorff, un coefficiente di attendibilità fra codificatori (Hughes \& Garrett, 1990) che misura la concordanza tra i codificatori per ciascuna categoria tenendo conto dell'accordo dovuto al caso e del numero di disaccordi (Neuendorf, 2002)

Una volta ottenuto un buon grado di attendibilità interpersonale ( $\alpha$ maggiore di .80) il codebook è stato utilizzato nella sua versione definitiva.

\subsubsection{Analisi quantitative}

Le categorie assegnate nella fase di codifica sono state riportate, assieme alle altre variabili quantitative dell'indagine ICCS, in un database, organizzate in una matrice in cui per riga fossero indicati i casi e per colonna la presenza/ assenza della specifica categoria. Questa organizzazione dei dati ha permesso di integrare informazioni di tipo qualitativo e quantitativo (Brewer \& Hunter, 2006). Per la parte quantitativa è stato impiegato il software SPAD versione 7.3. L'analisi ha consentito di verificare la presenza di pattern specifici attraverso la costruzione di uno strumento sintetico di visualizzazione, la caratterizzazione, che ha permesso di individuare le relazioni di ciascuna delle categorie di una variabile di interesse, di tipo continuo o nominale (Lebart, Morineau, \& Piron, 1995). Le caratterizzazioni si basano su dei confronti delle percentuali all'interno della classe con le percentuali ottenute sull'insieme degli elementi da classificare. Una volta definita la variabile da caratterizzare, è possibile leggerla in relazione a tutti i suoi elementi caratterizzanti. Una modalità viene considerata come caratteristica della classe se la sua presenza all'interno del gruppo è significativamente superiore a quella attesa. La variabile caratterizzante scelta è stato l'indicatore socioeconomico (SES), le modalità da caratterizzare sono state le categorie definite nel codebook.

\section{Risultati}

L'analisi esplorativa condotta sul campione di metafore ha permesso in prima battuta di costruire un codebook dei concetti chiave rintracciati attraverso 
l'analisi del contenuto. Il codebook racchiude le informazioni necessarie per una giusta allocazione delle categorie alla metafora, assicurando al contempo un alto grado di affidabilità (Neuendorf, 2002). Il codebook costituisce uno strumento informativo ed esemplificativo e assicura replicabilità alla ricerca. La tabella successiva (Tabella 2) riporta il codebook utilizzato per questo studio. Al fine di poter eseguire le successive analisi statistiche, sono state prese in considerazione le categorie con frequenza superiore a 20. Il codebook riportato in tabella è quello ottenuto a valle della codifica esplorativa, dopo la valutazione formativa dell'attendibilità.

Il "nome della categoria» racchiude il senso della categoria stessa ed è stato attribuito al termine del processo ricorsivo di attribuzione. La «definizione» consente di individuare i confini semantici della categoria, modulandone la distanza e fornendo una esplicitazione formale del suo contenuto. $\mathrm{La}$ «regola di codifica» include una definizione operativa della categoria che esplicita le tipologie di metafore da includere in quel gruppo. L'approccio è stato di tipo induttivo: sono state le precedenti codifiche, infatti, ad ampliare e a modulare i principi di applicazione. Le regole di codifica includono anche una descrizione dei casi più complessi e ambigui in modo da facilitare il codificatore nel processo di attribuzione. L'ultima colonna, infine, contiene una serie di esempi tratti dalle risposte date dagli studenti trascritte verbatim, riportando le metafore più frequenti e significative.

La Tabella 3 riporta la frequenza delle categorie individuate nelle metafore prodotte dagli studenti. La frequenza più alta è quella rappresentata dalla categoria "persone uguali a noi», pari a 127 , seguono «esseri viventi o cose fragili» ed «esseri viventi o cose sgradite», con frequenza pari rispettivamente a 113 e a 101. Le frequenze più basse, ma comunque maggiori di 20, sono invece costituite da "esseri viventi o cose eteree, senza consistenza" (frequen$\mathrm{za}=22)$ e "persone con i nostri stessi diritti, le nostre stesse opportunità» $($ frequenza $=21)$.

La Tabella 4 illustra le categorie caratterizzate rispetto allo status socioeconomico (SES). Come si evince dalla tabella, solo alcune categorie e non altre sono in relazione con il SES. In particolare, le categorie «esseri viventi o cose maltrattate, in pericolo», "una risorsa che arricchisce», "persone, animali o cose in cerca di benessere, di miglioramento" hanno evidenziato una relazione di segno positivo con il SES. Al contrario, la categoria «persone uguali a noi» ha mostrato una relazione di segno negativo. All'aumentare del SES quindi, l'immagine dell'immigrato è risultata maggiormente connotata con caratteristiche riconducibili all'essere maltrattato, bisognoso di miglioramento e opportunità, ma anche al costituire una risorsa per la società. $\mathrm{Al}$ tempo stesso, la relazione tra SES e uguaglianza ha assunto un andamento opposto: all'aumentare del SES gli immigrati sono meno persone uguali a noi. 
Tabella 2. - Il codebook che racchiude le categorie: nome della categoria, definizione, regola di codifica ed esempi di testi.

\begin{tabular}{|c|c|c|c|}
\hline $\begin{array}{c}\text { Nome } \\
\text { DELLA } \\
\text { CATEGORIA }\end{array}$ & Definizione & Regola Di CODIFICA & ESEMPI DI TESTI \\
\hline $\begin{array}{l}\text { Persone } \\
\text { uguali } \\
\text { a noi. }\end{array}$ & $\begin{array}{l}\text { Qualcuno che non } \\
\text { differisce da altro } \\
\text { o da altri, } \\
\text { sia in termini } \\
\text { complessivi } \\
\text { o per determinate } \\
\text { caratteristiche. }\end{array}$ & $\begin{array}{l}\text { Si fa riferimento a metafore che indicano } \\
\text { parità, stesse caratteristiche, stesse qualità } \\
\text { tra immigrati e «noi». Include le metafore } \\
\text { «sono come noi», "uguali a noi», "tutti noi» } \\
\text { e in generale le metafore in cu c'è un chiaro } \\
\text { riferimento di uguaglianza / non } \\
\text { differenziazione rispetto al gruppo «noi». } \\
\text { Sono incluse in questa categoria anche } \\
\text { le metafore come «amici» e «fratelli», } \\
\text { in generale quindi quelle che presuppongono } \\
\text { una relazione forte con il «noi». Non sono } \\
\text { invece incluse le metafore che fanno } \\
\text { un riferimento esplicito all'oggetto } \\
\text { dell'uguaglianza («sono uguali nei diritti») } \\
\text { che vanno codificate nella categoria «Persone } \\
\text { con i nostri stessi diritti, } \\
\text { le nostre stesse opportunità». Non sono incluse } \\
\text { le metafore che individuano una caratteristica } \\
\text { di differenza assieme all'uguaglianza («sono } \\
\text { come noi, ma di colore diverso») che vanno } \\
\text { codificate in «Persone che assomigliano a noi, } \\
\text { ma non in tutto». }\end{array}$ & $\begin{array}{l}\text { - Noi, perché siamo } \\
\text { tutti uguali. } \\
\text { - Sono come amici. } \\
\text { - Fratelli. }\end{array}$ \\
\hline $\begin{array}{l}\text { Esseri viventi } \\
\text { o cose fragili. }\end{array}$ & $\begin{array}{l}\text { Qualcuno/qualcosa } \\
\text { che è privo di difesa, } \\
\text { di protezione. } \\
\text { Si riferisce a esseri } \\
\text { viventi/non viventi } \\
\text { delicati, deboli. } \\
\text { Nella definizione } \\
\text { si include anche } \\
\text { chi è smarrito, } \\
\text { sperduto. }\end{array}$ & $\begin{array}{l}\text { Si fa riferimento a persone, animali, cose } \\
\text { in situazioni vulnerabili, indifesi, senza } \\
\text { protezione. Spesso riconducibili a bambini, } \\
\text { cuccioli, uccelli. Include situazioni, elementi } \\
\text { che esprimono qualcosa di delicato, instabile, } \\
\text { che può rompersi/corrompersi. Comprende } \\
\text { metafore che esprimono situazioni, stati } \\
\text { d'animo di smarrimento, di disorientamento, } \\
\text { di turbamento. Associate spesso alla perdita } \\
\text { di casa, al lasciare il nido, all'essere fuori } \\
\text { dal proprio contesto di riferimento. }\end{array}$ & $\begin{array}{l}\text { - Persone senza tetto. } \\
\text { - Oggetti che vengono } \\
\text { sbarcati in Italia. } \\
\text { - Sono come } \\
\text { un cucciolo } \\
\text { senza mamma. }\end{array}$ \\
\hline $\begin{array}{l}\text { Esseri viventi } \\
\text { o cose } \\
\text { sgradite. }\end{array}$ & $\begin{array}{l}\text { Qualcuno/qualcosa } \\
\text { di non desiderato, } \\
\text { di non gradito } \\
\text { che non risponde } \\
\text { alle aspettative. } \\
\text { Nella definizione } \\
\text { si include anche } \\
\text { chi è sporco, } \\
\text { non pulito. }\end{array}$ & $\begin{array}{l}\text { Si fa riferimento a metafore che includono } \\
\text { situazioni, persone, cose sgradite, che non } \\
\text { si desiderano. Tipicamente persone o animali } \\
\text { che disturbano, che possono provocare } \\
\text { un disagio, una situazione che si accetta } \\
\text { malvolentieri, qualcosa di troppo, che } \\
\text { provocano sensazioni sgradevoli. Include } \\
\text { similitudini che contengono esplicitamente } \\
\text { la parola «fastidio» oppure insiemi di parole } \\
\text { che richiamano una situazione che mal } \\
\text { si sopporta «rompiscatole», "polvere». Questa } \\
\text { categoria include anche quell'insieme } \\
\text { di animali (per lo più insetti) noti } \\
\text { comunemente per il loro essere fastidiosi } \\
\text { (come le zanzare, le pulci, le zecche). }\end{array}$ & $\begin{array}{l}\text { - Le pulci sulla schiena } \\
\text { di un cane. } \\
\text { - Gli stranieri che } \\
\text { noi Italiani } \\
\text { non vogliamo. } \\
\text { - Una macchia } \\
\text { indelebile } \\
\text { sulla camicia. }\end{array}$ \\
\hline
\end{tabular}


Nome

Definizione

REgOLA DI CODIFICA

ESEMPI DI TESTI

DELLA

CATEGORIA

\begin{tabular}{ll}
\hline Esseri viventi & Qualcuno/qualcosa \\
o cose & che si muove, \\
che & che si sposta. Indica \\
si spostano. & sia un movimento \\
& generico, \\
& senza particolari \\
& connotazioni \\
& o tratti; \\
& sia un movimento \\
& continuo, \\
& migratorio.
\end{tabular}

Si fa riferimento all'atto del muoversi, dello spostarsi, di persone, animali o cose che viaggiano e si spostano. L'azione di spostamento può essere temporanea o definitiva. Nella metafora vi è un riferimento ad una accezione di permanenza, di stabilità, spesso accompagnata dall'avverbio «sempre» o situazioni in cui il movimento non si arresta, è continuo. In questa categoria rientrano anche le metafore che si riferiscono a migrazioni o vagabondaggio, nomadismo.

\begin{tabular}{ll}
\hline Esseri viventi & Qualcuno/qualcosa \\
o cose & che differisce, \\
diverse & che non è uguale, \\
da noi. & che è di natura \\
& diversa.
\end{tabular}

Si fa riferimento a metafore che presuppongono una dimensione di identità vista come altra rispetto alla propria. Identifica una distanza sostanziale dall'altro, una distinzione tra «noi» e «loro». Include metafore che sottolineano questa diversità. Si fa inoltre riferimento a situazioni/persone/ animali/cose che sono al di fuori del loro contesto abituale, ad esempio estranei ad un gruppo di simili o stranieri.

\begin{tabular}{ll}
\hline $\begin{array}{l}\text { Esseri viventi } \\
\text { o cose }\end{array}$ & $\begin{array}{l}\text { Qualcuno/qualcosa } \\
\text { che costituisce } \\
\text { minacciose, }\end{array}$ \\
pericolose. & un pericolo, \\
& uninaccia che \\
& può preannunciare \\
& mon danno fisico, \\
& di male.
\end{tabular}

Si fa riferimento a persone/animali o situazioni considerate come un pericolo imminente per il proprio benessere fisico, materiale o immateriale. Sono metafore spesso riconducibili al mondo del lavoro, come "persone che ci portano via il lavoro", "che ci vogliono fregare», «invasori». Include situazioni, persone, animali che provocano un danno (o anche una sottrazione). Include anche metafore che descrivono situazioni di sottrazione indebita, di violazione e/o appropriazione di spazi, oggetti, risorse.

Si fa riferimento a cose concrete o astratte,
persone, animali che portano ad un arricchimento a livello materiale, culturale, intangibile. Include metafore che esplicitamente presentano un «arricchimento» e paragoni che fanno riferimento agli immigrati come una «risorsa», come un elemento in grado di dare nuove potenzialità e vantaggi al paese "luce nuova», «nuova scoperta», «tesoro».
- Delle macchine che viaggiano.

- Gli uccelli migratori.

- Dei viandanti in cerca di fortuna.
- Della carne messa nel cestino della frutta.

- Persone venute da un altro mondo.

- Sconosciuti.

\begin{tabular}{ll}
\hline $\begin{array}{l}\text { Una risorsa } \\
\text { che }\end{array}$ & $\begin{array}{l}\text { Qualcuno/qualcosa } \\
\text { arricchisce. }\end{array}$ \\
& che arricchisce, \\
& complete più \\
& che soddisfa \\
& un bisogno \\
& o una necessità, \\
& che rappresenta \\
& una risorsa.
\end{tabular}


Nome

Definizione

RegOLA DI CODIFICA

ESEMPI DI TESTI

DELLA

CATEGORIA

\begin{tabular}{ll}
\hline Esseri viventi & Qualcuno/qualcosa \\
o cose molto & che è costituito \\
numerosi. & da molti elementi, \\
& molte parti, \\
& che si presenta \\
& in gran numero.
\end{tabular}

Si fa riferimento a metafore che esprimono

- Le formiche non una grande quantità, sia di cose, di animali finiscono mai. o di persone. La metafora deve contenere - Pezzi di un puzzle. un riferimento alla quantità come ad esempio • I funghi spuntano «molto», «tanto» e i relativi superlativi. Può dappertutto. anche contenere similitudini che esprimono una quantità elevata senza per forza contenere le parole tanto e molto come ad esempio «sono infiniti», «non finiscono mai».

\begin{tabular}{ll}
\hline Persone & Qualcuno che non \\
normali, & differisce dall'altro \\
come tutti. & (in termini \\
& più generici rispetto \\
& a «uguali a noi»), \\
& che non è al di fuori \\
& della norma, \\
& che è comune.
\end{tabular}

Si fa riferimento a metafore che indicano parità, stesse caratteristiche, stesse qualità tra persone in maniera generica, senza un paragone con il gruppo del «noi». Individua quindi paragoni che fanno riferimento a uguaglianza / non differenziazione, ma senza un chiaro confronto con «noi». Include metafore che fanno riferimento all'essere persone «comuni», «normali», "come gli altri».

\begin{tabular}{ll}
\hline Una novità. & Qualcuno/qualcosa \\
& che costituisce \\
& una novità, che \\
& è iniziato/accaduto \\
& di recente, che prima \\
& non c'era.
\end{tabular}

Esseri viventi Qualcuno/qualcosa o cose maltrattate, che è trattato male, in pericolo. sia a parole o con atti violenti. Indica anche chi può essere in pericolo.

Si fa riferimento a metafore che individuano qualcosa di nuovo, di inaspettato, una novità rispetto ad una situazione pre-esistente.

Si fa riferimento a similitudini che si riferiscono a bambini/animali appena nati, che rinascono.
- Nuove persone.

- Delle novità.

- Neonati.
- Persone normali.

- Tutti gli altri.

- Persone qualunque.
Si fa riferimento a metafore che presentano situazioni, persone, animali o cose che vengono trattate male, che sono in una situazione potenzialmente pericolosa e che sono minacciati. Comprende metafore che parlano di animali in fuga e minacciati da altri animali.
- I cani, spesso maltrattati.

- Delle boe abbandonate in mezzo al mare e prese a calci dalle onde.

- Un gregge in fuga da un animale feroce.

\begin{tabular}{|c|c|c|c|}
\hline $\begin{array}{l}\text { Esseri viventi } \\
\text { che ci } \\
\text { sfruttano, } \\
\text { opportunisti. }\end{array}$ & $\begin{array}{l}\text { Qualcuno } \\
\text { che si adegua } \\
\text { di volta in volta } \\
\text { alle circostanze, } \\
\text { cercando } \\
\text { di sfruttarle } \\
\text { a proprio vantaggio. }\end{array}$ & $\begin{array}{l}\text { Si fa riferimento a metafore che indicano } \\
\text { comportamenti di tipo interessato, } \\
\text { che portano ad un vantaggio, } \\
\text { ad una comodità per chi li perpetua. Inoltre } \\
\text { si fa riferimento a metafore che includono } \\
\text { per lo più animali (ma anche persone) che } \\
\text { per sopravvivere dipendono da un'altra } \\
\text { persona/animale. Comunemente i pidocchi, } \\
\text { le zecche, le pulci. Si può trovare anche } \\
\text { nella sua forma più generale di parassita. Si fa } \\
\text { riferimento anche a paragoni riconducibili } \\
\text { a situazioni, animali che ricordano } \\
\text { una condizione di dipendenza, di legame. }\end{array}$ & $\begin{array}{l}\text { - Opportunisti. } \\
\text { - Le sanguisughe } \\
\text { che tolgono lavoro } \\
\text { agli altri. } \\
\text { - Il verme nella mela. }\end{array}$ \\
\hline
\end{tabular}


Nome

Definizione

Regola DI CODIFICA

ESEMPI DI TESTI

DELLA

CATEGORIA

\begin{tabular}{|c|c|c|c|}
\hline $\begin{array}{l}\text { Persone, } \\
\text { animali } \\
\text { o cose } \\
\text { bisognosi } \\
\text { di aiuto, } \\
\text { di cure. }\end{array}$ & $\begin{array}{l}\text { Qualcuno/qualcosa } \\
\text { che ha bisogno } \\
\text { di aiuto, a cui manca } \\
\text { una cosa necessaria. }\end{array}$ & $\begin{array}{l}\text { Si fa riferimento a metafore che evidenziano } \\
\text { situazioni di bisogno, per lo più riferite } \\
\text { a bisogno di aiuto o di cure. Le metafore } \\
\text { possono fare esplicitamente riferimento } \\
\text { alla protezione, all'essere protetti, } \\
\text { alla necessità di cure, all'aiuto. Di solito sono } \\
\text { riferite a persone bisognose, a bambini, } \\
\text { fratelli, cani bastonati, cuccioli. }\end{array}$ & $\begin{array}{l}\text { - Persone bisognose. } \\
\text { - Dei bambini che } \\
\text { hanno bisogno } \\
\text { di cure. } \\
\text { - Persone da aiutare. }\end{array}$ \\
\hline $\begin{array}{l}\text { Persone, } \\
\text { animali } \\
\text { o cose } \\
\text { in cerca } \\
\text { di benessere, } \\
\text { di migliora- } \\
\text { mento. }\end{array}$ & $\begin{array}{l}\text { Qualcuno/qualcosa } \\
\text { che è alla ricerca } \\
\text { di una situazione } \\
\text { maggiormente } \\
\text { favorevole, } \\
\text { di benessere, } \\
\text { una situazione di vita } \\
\text { migliore. }\end{array}$ & $\begin{array}{l}\text { Si fa riferimento a situazioni } \\
\text { di miglioramento, di cambiamento } \\
\text { in positivo nella vita di persone/animali/cose } \\
\text { o di una aspettativa di crescita e sviluppo. } \\
\text { Tipicamente relativi a persone che lasciano } \\
\text { il paese in cerca di condizioni migliori, } \\
\text { di un lavoro, di fortuna. Legato a situazioni } \\
\text { volte alla ricerca/ottenimento/mantenimento } \\
\text { di benessere. Spesso inteso come ricerca } \\
\text { di miglioramento/sviluppo/crescita. }\end{array}$ & $\begin{array}{l}\text { - Una borsa piena } \\
\text { di speranze, desideri } \\
\text { e futuro. } \\
\text { - Delle persone } \\
\text { che cercano } \\
\text { condizioni di vita } \\
\text { migliori. } \\
\text { - Gli uccelli, lasciano } \\
\text { il loro paese in cerca } \\
\text { di fortuna. }\end{array}$ \\
\hline $\begin{array}{l}\text { Esseri viventi } \\
\text { lavoratori, } \\
\text { operosi. }\end{array}$ & $\begin{array}{l}\text { Qualcuno } \\
\text { di industrioso, } \\
\text { che si impegna } \\
\text { nel proprio } \\
\text { lavoro/vita. }\end{array}$ & $\begin{array}{l}\text { Si fa riferimento a metafore riconducibili } \\
\text { a laboriosità, solerzia. Include similitudini } \\
\text { che fanno riferimento al lavoro, } \\
\text { o agli immigrati come forza-lavoro. Include } \\
\text { anche animali comunemente associati } \\
\text { a qualità di operosità o alacrità, come api } \\
\text { o formiche. }\end{array}$ & $\begin{array}{l}\text { - Le formiche. } \\
\text { - Le api. } \\
\text { - Lavoratori. }\end{array}$ \\
\hline
\end{tabular}

\begin{tabular}{|c|c|c|c|}
\hline $\begin{array}{l}\text { Persone, } \\
\text { animali } \\
\text { o cose } \\
\text { senza casa, } \\
\text { senza un nido. }\end{array}$ & $\begin{array}{l}\text { Qualcuno/qualcosa } \\
\text { che non ha una casa } \\
\text { in cui stare. }\end{array}$ & $\begin{array}{l}\text { Si fa riferimento a metafore che evidenziano } \\
\text { situazioni di bisogno di una casa o di un nido } \\
\text { (nel caso di animali). Include anche metafore } \\
\text { del mondo vegetale che fanno riferimento } \\
\text { alla mancanza di radici (quindi di una casa } \\
\text { anche dal punto di vista affettivo e valoriale). }\end{array}$ & $\begin{array}{l}\text { - Persone senza tetto; } \\
\text { - Uccelli senza nido; } \\
\text { - Le nuvole, non } \\
\text { hanno una meta, né } \\
\text { una casa. }\end{array}$ \\
\hline $\begin{array}{l}\text { Persone che } \\
\text { assomigliano } \\
\text { a noi, ma } \\
\text { non in tutto. }\end{array}$ & $\begin{array}{l}\text { Qualcuno che } \\
\text { assomiglia ad un altro } \\
\text { o ad altri per alcune } \\
\text { caratteristiche, ma } \\
\text { che differisce per } \\
\text { qualcosa, che presenta } \\
\text { elementi di diversità, } \\
\text { che si distingue. }\end{array}$ & $\begin{array}{l}\text { Si fa riferimento a metafore che indicano } \\
\text { parità, stesse caratteristiche, stesse qualità } \\
\text { tra persone, ma che allo stesso tempo } \\
\text { presentano una differenza, spesso introdotta } \\
\text { dalla congiunzione «ma». Indicano quindi } \\
\text { una situazione di non totale accordo, in cui } \\
\text { da un lato si evidenzia l'uguaglianza rispetto } \\
\text { al «noi», ma dall'altro si sottolinea } \\
\text { una differenza. }\end{array}$ & $\begin{array}{l}\text { - Fratelli, che però } \\
\text { sono lontani da noi. } \\
\text { - Noi, però } \\
\text { sono sfortunati. } \\
\text { - Noi solo } \\
\text { di colorazione } \\
\text { diversa. }\end{array}$ \\
\hline $\begin{array}{l}\text { Persone, } \\
\text { animali } \\
\text { o cose } \\
\text { buone, } \\
\text { gentili. }\end{array}$ & $\begin{array}{l}\text { Qualcuno/qualcosa } \\
\text { che tende al bene, } \\
\text { onesto, positivo. } \\
\text { Include anche chi } \\
\text { è mansueto, bonario. }\end{array}$ & $\begin{array}{l}\text { Si fa riferimento a metafore che si collegano } \\
\text { ad un senso di mansuetudine, di bontà } \\
\text { d'animo, di benevolenza, gentilezza. Include } \\
\text { similitudini che fanno riferimento } \\
\text { al carattere innocente degli immigrati. }\end{array}$ & $\begin{array}{l}\text { - Brave persone. } \\
\text { - Il pane. } \\
\text { - Dei poveri innocenti. }\end{array}$ \\
\hline
\end{tabular}




\section{Nome}

Definizione

Regola DI CODIFICA

ESEMPI DI TESTI

DELLA

CATEGORIA

\begin{tabular}{|c|c|c|c|}
\hline $\begin{array}{l}\text { Esseri viventi } \\
\text { o cose eteree, } \\
\text { senza } \\
\text { consistenza. }\end{array}$ & $\begin{array}{l}\text { Qualcuno/qualcosa } \\
\text { che evapora } \\
\text { rapidamente, che } \\
\text { è inconsistente, } \\
\text { che non ha solidità. }\end{array}$ & $\begin{array}{l}\text { Si fa riferimento a metafore che comunicano } \\
\text { più o meno esplicitamente un senso } \\
\text { di inconsistenza, di immaterialità, } \\
\text { di leggerezza, per lo più riconducibili } \\
\text { ad agenti atmosferici o animali che hanno } \\
\text { tratti di leggerezza o sono eterei. Molto spesso } \\
\text { in metafore che fanno riferimento a nuvole, } \\
\text { pioggia, vento, farfalle, aria. }\end{array}$ & $\begin{array}{l}\text { - Piume al vento. } \\
\text { - La pioggia. } \\
\text { - Una ventata d'aria } \\
\text { fresca. }\end{array}$ \\
\hline $\begin{array}{l}\text { Persone con } \\
\text { i nostri stessi } \\
\text { diritti, } \\
\text { le nostre } \\
\text { stesse } \\
\text { opportunità. }\end{array}$ & $\begin{array}{l}\text { Qualcuno che non } \\
\text { si differenzia } \\
\text { da un altro o da altri } \\
\text { per un determinato } \\
\text { tratto, per una } \\
\text { caratteristica specifica, } \\
\text { in particolare riferita } \\
\text { alla sfera dei diritti } \\
\text { e delle opportunità. }\end{array}$ & $\begin{array}{l}\text { Si fa riferimento a metafore che indicano } \\
\text { parità, stesse caratteristiche, stesse qualità } \\
\text { tra persone per una specifica caratteristica, } \\
\text { come ad esempio il rispetto degli stessi } \\
\text { diritti o doveri. }\end{array}$ & $\begin{array}{l}\text { - Persone con gli stessi } \\
\text { diritti e gli stessi } \\
\text { doveri. } \\
\text { - Delle persone } \\
\text { cittadine. } \\
\text { - Noi, con gli stessi } \\
\text { diritti. }\end{array}$ \\
\hline
\end{tabular}

Tabella 3. - La frequenza delle categorie (maggiore di 20).

\begin{tabular}{lc}
\hline \multicolumn{1}{c}{ CATEGORIE } & FREQUENZA > 20 \\
\hline Persone uguali a noi & 127 \\
Esseri viventi o cose fragili & 113 \\
Esseri viventi o cose sgradite & 101 \\
Esseri viventi o cose che si spostano & 91 \\
Esseri viventi o cose diverse da noi & 86 \\
Esseri viventi o cose minacciose, pericolose & 75 \\
Una risorsa che arricchisce & 74 \\
Esseri viventi o cose molto numerosi & 67 \\
Persone normali, come tutti & 65 \\
Una novità & 44 \\
Esseri viventi o cose maltrattate, in pericolo & 38 \\
Esseri viventi che ci sfruttano, opportunisti & 35 \\
Persone, animali o cose bisognosi di aiuto, di cure & 34 \\
Persone, animali o cose in cerca di benessere, di miglioramento & 33 \\
Esseri viventi lavoratori, operosi & 30 \\
Persone, animali o cose senza casa, senza un nido & 29 \\
Persone che assomigliano a noi, ma non in tutto & 26 \\
Persone, animali o cose buone, gentili & 25 \\
Esseri viventi o cose eteree, senza consistenza & 22 \\
Persone con i nostri stessi diritti, le nostre stesse opportunità & 21 \\
\hline
\end{tabular}


Tabella 4. - Le categorie caratterizzate

rispetto allo status socioeconomico familiare di provenienza.

\begin{tabular}{|c|c|c|c|c|}
\hline Categoria & $\begin{array}{c}\text { DIREZIONE } \\
\text { DELLA } \\
\text { DIFFERENZA }\end{array}$ & Media & $\begin{array}{l}\text { DEVIAZIONE } \\
\text { STANDARD }\end{array}$ & Probabilità \\
\hline $\begin{array}{l}\text { Esseri viventi o cose maltrattate, } \\
\text { in pericolo }\end{array}$ & + & 0,38 & 1,22 & 0,004 \\
\hline Una risorsa che arricchisce & + & 0,22 & 1,02 & 0,007 \\
\hline Persone uguali a noi & - & $-0,04$ & 1,02 & 0,014 \\
\hline $\begin{array}{l}\text { Persone, animali o cose in cerca } \\
\text { di benessere, di miglioramento }\end{array}$ & + & 0,30 & 1,16 & 0,019 \\
\hline
\end{tabular}

Nota. Direzione della differenza: indica se per i soggetti che hanno risposte codificate nella categoria in oggetto la media del SES è significativamente più alta $(+)$ o più bassa $(-)$ della media del campione generale. La scala del SES è standardizzata con media 0 e deviazione standard pari a 1.

\section{Discussione}

Lo studio ha cercato di ricostruire le rappresentazioni che gli studenti hanno degli immigrati attraverso l'utilizzo di metodologie di ricerca mista, in grado di tenere conto della ricchezza e della complessità delle rappresentazioni proposte. L'esame degli atteggiamenti rispetto all'immigrazione, proprio perché riferito ad un tema rilevante, influenzato fortemente dal contesto e dalla desiderabilità sociale, si presta infatti, quando indagato con metodologie tradizionali, a restituire forme cristallizzate e monodimensionali di ciò che pensano gli studenti.

I risultati delle scale ICCS sugli atteggiamenti dei giovani verso i diritti degli immigrati riportati nell'introduzione porterebbero a pensare che anche le immagini restituite dalle metafore seguano una linea di giudizio per lo più negativa, basata su stereotipi e pregiudizi. Effettivamente una delle dimensioni che è emersa dall'analisi del contenuto è proprio questa: categorie come "esseri viventi o cose minacciose, pericolose», «esseri viventi o cose sgradite», «esseri viventi che ci sfruttano, opportunisti» si rifanno ad una visione dell'immigrato percepito in modo ostile, appunto come una minaccia. Queste immagini raccontano la propensione degli individui a costruire dei gruppi (in-group e out-group) e a identificarsi come membri di uno specifico gruppo e non di un altro, portando così alla formazione delle identità sociali (Tajfel \& Turner, 1979; Tajfel, 1982) Per Tajfel (1982) la creazione dei gruppi av- 
viene in base a criteri interni o esterni, basati sull'identificazione di sé come membro di quello specifico insieme di individui e su elementi sia cognitivi che affettivi. L'identificazione nei gruppi guida lo sviluppo di processi di tipo valutativo tesi a favorire i membri del proprio gruppo (in-group favoritism) e a non favorire il gruppo esterno (out-group derogation).

Le categorie "esseri viventi che ci sfruttano, opportunisti», "esseri viventi o cose molto numerosi», "esseri viventi o cose sgradite», richiamano un'immagine dello straniero come una minaccia, reale o percepita, rispetto agli interessi e ai valori dell'in-group (Sherif, 1966; Blalock, 1967; Stephan, Ybarra, \& Bachman, 1999). La dimensione valutativa basata su immagini non favorevoli dell'out-group può portare ad atteggiamenti di esclusione, pregiudizio, stereotipia (Stephan \& Stephan, 2000) in grado di guidare poi comportamenti ed emozioni (Quillian, 1995). Nelle metafore si parlava degli immigrati "come spazzatura», "come pidocchi», "come dei ladri in casa mia», "come il verme nella mela", "come invasori", tutti richiami ad un mondo concettuale negativamente stereotipato, in cui l'immigrato viene percepito come sporco, criminale e cattivo.

Una lettura delle categorie che si riconduca esclusivamente alla contrapposizione tra in-group e out-group non sarebbe però esaustiva. Le analisi hanno mostrato, infatti, delle aree semantiche che si discostano da questa interpretazione, implicando processi di acculturazione non per forza conflittuali (Berry, 2005) e che complessificano la relazione dualistica «noi»-»loro». Un tema frequente è stato ad esempio quello che si è mosso sull'asse dell'uguaglianza. Le similitudini in questione hanno descritto un mondo in cui gli immigrati sono "persone uguali a noi» e hanno le stesse opportunità e gli stessi diritti degli Italiani. Le metafore hanno descritto gli immigrati «come fratelli», "come tutti gli altri», "come cittadini», «come noi, ma sfortunati» richiamando valutazioni non solo positive, ma centrate su temi civici, di tolleranza, di inclusione e partecipazione. Una possibile chiave di lettura per questi concetti sono le ricerche in ambito multiculturale. Ad esempio il modello proposto da Berry (Berry, 1997; Schwartz \& Zamboanga, 2008) individua diverse strategie interculturali che colgono la doppia spinta al mantenimento della propria identità culturale da un lato e alla relazione tra i gruppi dall'altro. Sulla base di queste due dimensioni, le strategie messe in campo sono essenzialmente quattro, di cui l'integrazione coniuga la tendenza ad entrare in contatto con il gruppo maggioritario mantenendo la cultura del paese d'origine, mentre al polo opposto, la marginalizzazione presuppone l'assenza di una relazione con entrambe le culture. Lo stimolo che è emerso dalle metafore centrate sull'uguaglianza sembra richiamare in particolare due delle dimensioni individuate da Berry, quella dell'assimilazione (o del melting pot se visto dal punto di vista dell'in-group) e quella 
dell'integrazione (o del multiculturalismo, sempre se si predilige il punto di vista dell' in-group). Le metafore centrate sull'uguaglianza colgono un aspetto di integrazione, di tolleranza e di reciproco interesse, in cui il proprio bagaglio culturale rimane inalterato. D'altro canto queste metafore sembrano invece far riferimento ad un progressivo assorbimento dell'out-group nel gruppo prevalente a scapito quindi delle proprie origini culturali (Portes $\&$ Zhou, 1993; Alba \& Nee, 1997; Portes, Fernandez-Kelly, \& Haller, 2005). Categorie come «esseri viventi lavoratori, operosi», «una risorsa che arricchisce», "persone, animali o cose buone, gentili» prediligono infatti una percezione dell'altro come risorsa, come qualcosa di utile alla propria società, di buono e innocuo, dando importanza alla sfera lavorativa nei processi di socializzazione e costruzione dell'identità sociale (Giovannini, Pintus, \& Rinaldi, 2007).

Un gruppo particolarmente consistente di metafore è quello che ha restituito un'immagine dell'immigrato come perduto, indifeso, fragile, costituito da persone, ma anche e soprattutto bambini e cuccioli, "smarriti", «abbandonati», "senza casa» o "in cerca di un futuro migliore». In questo gruppo di similitudini, la componente che è sembrata predominante è stata quella cognitiva-affettiva dell'empatia. Secondo Batson (2009), gli individui sono motivati ad alleviare le difficoltà e le sofferenze degli altri e ad attivare comportamenti di aiuto quando ne riconoscono le esperienze emozionali e ne percepiscono le emozioni e le sensazioni. L'empatia è un costrutto multidimensionale composto principalmente da una componente cognitiva e da una emotiva (e.g., Stephan \& Stephan, 2001; Dziobek et al., 2008). La componente cognitiva è centrata sulla capacità dellindividuo di assumere il ruolo dell'altro e il suo punto di vista, deducendo i suoi stati mentali. Secondo Stephan e Stephan (2001), questa dimensione dell'empatia riduce il grado di intelligibilità e aumenta il livello di comprensibilità, limitando così sentimenti di paura e incertezza. La seconda componente si rifa alle risposte emotive verso gli stati emotivi di un'altra persona e comprende sentimenti di compassione e di preoccupazione per le sofferenze dell'altro. Diverse ricerche hanno mostrato l'importanza dell'empatia come effetto di mediazione per la riduzione del pregiudizio nelle relazioni intergruppo (e.g., Batson et al., 1997; Finlay \& Stephan, 2000; Pedersen et al., 2004; Voci \& Hewstone, 2007; Dovidio et al., 2010). Le influenze di tipo emotivo e gli schemi mentali giocano quindi un ruolo importante nella percezione (e.g., Bonaiuto, Biasi, \& Giannini, 2008; Biasi, Chiappetta Cajola, \& Bonaiuto, 2010): le metafore di questo gruppo richiamano una visione emozionale e quasi compassionevole dell'altro, in cui l'immigrato viene percepito come soggetto debole, bisognoso di cura e assistenza (Mezzadra, 2006; Buzzi, Cavalli, \& De Lillo, 2007). Una percezione di questo tipo, può contribuire a ridurre 
la formazione di stereotipi e dare l'impulso a comportamenti volti a ridurre discriminazioni e ingiustizie (Voci \& Hewstone, 2007).

Infine è presente un gruppo di paragoni riconducibili all'essere in movimento, in modo costante, ricorsivo («sono come uccelli migratori») o legato allo spostarsi da un luogo ad un altro ("sono come animali che si spostano»). La categoria "esseri viventi o cose che si spostano» si riferisce a metafore tautologiche, che non aggiungono nulla di più rispetto al dominio target della metafora stessa e che sono poco salienti rispetto al mondo concettuale che richiamano.

In generale, le immagini che sono risultate dall'analisi non hanno solo una forte valenza evocativa, ma forniscono informazioni preziose sugli atteggiamenti degli studenti rispetto all'immigrato: rappresentare l'altro come una sanguisuga, come un invasore, come immondizia, è diverso da immaginarlo come un cane abbandonato, un bambino sperduto o una pecora tra i lupi, così come è ancora diverso vederlo come un libro, come un cesto pieno di frutta, come un fratello. Al di là della connotazione positivo/negativo, è risultato interessante cogliere le differenze, far emergere grazie ad un singolo stimolo la popolazione composita di questi mondi concettuali.

Se dal punto di vista dei contenuti delle immagini mentali questo studio ha contribuito ad arricchire il quadro rispetto all'immigrazione, anche dal punto di vista metodologico la ricerca ha presentato alcuni spunti importanti. L'utilizzo di una metodologia di ricerca mista ha infatti permesso di mettere in relazione un approccio più induttivo, utilizzato nel processo ricorsivo di codifica e poi nell' analisi del contenuto, con uno tipicamente deduttivo espresso dalla lettura delle categorie ottenute in relazione con una variabile di interesse quantitativa (il SES). I risultati hanno mostrato che ad un maggiore livello di istruzione e di status occupazionale dei genitori, nonché in presenza di più libri a casa, le immagini tendono ad essere riconducibili ad una dimensione emotiva, legata al bisogno, ad un moto di vicinanza, ad un compatimento della condizione dell'altro. Al tempo stesso però gli immigrati sono stati percepiti non come persone uguali a noi, ma come qualcosa d'altro, un gruppo caratterizzato da attributi legati alla loro condizione di svantaggio e da un sistema di opportunità più povero, tutt'altro che facilitante. A livelli di SES più bassi, invece, la percezione è apparsa essere quella degli immigrati come persone uguali a noi, mentre si è persa la connotazione empatica vista per i livelli di status socioeconomico più alti.

In conclusione i risultati di questo studio hanno messo in luce come la rappresentazione del tema sia complessa: la varietà e il numero di immagini è stato infatti consistente e ha spaziato tra entità concettuali anche molto distanti tra loro. Il concentrarsi sui contenuti degli atteggiamenti più che descriverne a priori la paternità in termini valutativi, ha consentito di ampliare la dimensio- 
ne semantica e di cogliere ambiti meno espliciti, difficilmente rintracciabili da strumenti puramente quantitativi. Le metodologie di ricerca mista utilizzate hanno presentato diversi vantaggi nell'applicazione a questo ambito di ricerca. In particolare, hanno consentito da un lato di scoprire dimensioni semantiche meno visibili all'analisi quantitativa; dall'altro di avere una valutazione affidabile delle dimensioni proposte, nonché la possibilità di testare le differenze tra studenti con strumenti statistici (Alivernini, Lucidi, \& Manganelli, 2008), aumentando la flessibilità delle analisi in base alle domande di ricerca. Le metafore hanno permesso di indagare dimensioni sensibili, difficili e di restituire un mondo più articolato, vivace e ricco di rappresentazioni. La ricerca ha permesso di individuare dimensioni semantiche nuove che hanno restituito una rappresentazione viva dei mondi concettuali dei giovani studenti. Lampiezza e la varietà di queste immagini possono costituire un contributo non solo di tipo metodologico, ma anche di tipo sociale ed educativo. L'attenzione ai contenuti più che ai processi fornisce elementi utili per comprendere gli oggetti che popolano le rappresentazioni dei giovani intervistati. Questi oggetti, come si è visto, non sono monodimensionali e restituiscono molte informazioni sulla natura delle relazioni intergruppo. Esplorare questi temi, a scuola e nella società in generale, può significare avere una conoscenza degli elementi che vanno a costituire le immagini sociali legate a giudizio e stereotipia e riflettere e lavorare su questi. La consapevolezza di questi contenuti può arricchire l'immagine dell'altro, restituirle la propria complessità e aiutare a rafforzare atteggiamenti di tolleranza, di confronto e di rispetto reciproco.

\section{RIFERIMENTI BIBLIOGRAFICI}

Alba, R., \& Nee, V. (1997). Rethinking assimilation theory for a new era of immigration. The International Migration Review, 31(4), 826-874, doi: 10.2307/2547416.

Alivernini, F. (2012). Mixed methods research on learning. In M. Seel (Ed.), Encyclopedia of the sciences of learning. New York: Springer, doi: 10.1007/978-14419-1428-6_846.

Alivernini, F., Cavicchiolo, E., Palmerio, L., \& Girelli, L. (2015). Representations of study and students' academic motivation. Procedia-Social and Behavioral Sciences, 205, 302-305, doi: 10.1016/j.sbspro.2015.09.086.

Alivernini, F., Lucidi, F., \& Manganelli, S. (2008). Assessment of academic motivation: A mixed methods study. International Journal of Multiple Research Approaches, 2(1), 71-82, doi: 10.5172/mra.455.2.1.71.

Alivernini, F., \& Manganelli, S. (2016). The Classmates Social Isolation Questionnaire: An initial validation. European Journal of Developmental Psychology, 13(2), 264-274, doi: 10.1080/17405629.2016.1152174. 
Allport, G. W. (1954). The nature of prejudice. Cambridge, MA: Perseus Books.

Barber, C., Torney-Purta, J., \& Fennelly, K. (2010). Adolescents' attitudes toward immigrants' rights and nationalism in 25 countries. In 4 th IEA International Research Conference (IRC-2010), Gothenburg (Sweden). Retrieved from: http://www.iea.nl/fileadmin/user_upload/IRC/IRC_2010/Papers/

IRC2010_Barber_Torney-Purta_etal.pdf

Batson, C. D. (2009). These things called empathy: Eight related but distinct phenomena. In J. Decety \& W. J. Ickes (Eds.), The social neuroscience of empathy (pp. 3-15). Cambridge, MA: MIT Press, doi: 10.7551/mitpress/9780262012973.003.0002.

Batson, C. D., Polycarpou, M. P., Harmon-Jones, E., Imhoff, H. J., Mitchener, E. C., Bednar, L. L., et al. (1997). Empathy and attitudes: Can feeling for a member of a stigmatized group improve feelings toward the group? Journal of Personality and Social Psychology, 72, 105-118, doi: http://dx.doi. org/10.1037/0022-3514.72.1.105.

Berry, J. W. (1997). Immigration, acculturation, and adaptation. Applied Psychology, 46(1), 5-34, doi: 10.1111 / j.1464-0597.1997.tb01087.x.

Berry, J. W. (2005). Acculturation: Living successfully in two cultures. International Journal of Intercultural Relations, 29(6), 697-712, doi: 10.1016/j.ijintrel. 2005.07.013.

Biasi, V., Chiappetta Cajola, L., \& Bonaiuto, P. (2010). Valutare la formazione degli schemi mentali nei disturbi dell'apprendimento. Journal of Educational, Cultural and Psychological Studies, 2, 117-138.

Blalock, H. M. (1967). Toward a theory of minority-group relations. New York: J. Wiley \& Sons, doi: 10.1016/j.ijintrel.2015.10.002.

Blumer, H. (1958). Race prejudice as a sense of group position. The Pacific Sociological Review, 1(1), 3-7, doi: 10.2307/1388607.

Bohner, G., \& Dickel, N. (2011). Attitudes and attitude change. Annual Review of Psychology, 62, 391-417, doi: 10.1146/annurev.psych.121208.131609.

Bonaiuto, P., Biasi, V., \& Giannini, A. M. (2008). Connections between phenomenological-experimental research and psychodynamic constructs. Teorie \& Modelli, 13(2/3), 197-216.

Brandini, S. (2015). A mixed methods analysis of students' views of politics. ProcediaSocialand Behavioral Sciences, 177,273-277, doi: 10.1016/j.sbspro.2015.02.333.

Brewer, J., \& Hunter, A. (2006). Foundations of multimethod research: Synthesizing styles. Thousand Oaks, CA - London: Sage, doi: http://dx.doi. org/10.4135/9781412984294.

Buzzi, C., Cavalli, A., \& De Lillo, A. (a cura di). (2007). Rapporto giovani. Sesta indagine dell'Istituto IARD sulla condizione giovani in Italia. Bologna: il Mulino.

Caro, D. H., \& Schulz, W. (2012). Ten hypotheses about tolerance toward minorities among Latin American adolescents. Citizenship, Social and Economics Education, 11(3), 213-234, doi: 10.2304/csee.2012.11.3.213. 
Castelli, L., De Amicis, L., Carnaghi, A., \& Arcuri, L. (2007). L'influenza dei processi intragruppo sul contatto intergruppi: il caso dei bambini in età prescolare. In R. Brown, D. Capozza, \& O. Licciardello (a cura di), Immigrazione, acculturazione, modalità di contatto (pp. 117-135). Milano: FrancoAngeli.

Cavicchiolo, E., Alivernini, F., \& Manganelli, S. (2015). Students' images of immigrants in Italy: First results from a national mixed methods study. Procedia-Social and Behavioral Sciences, 205, 163-167, doi: 10.1016/j.sbspro.2015.09.049.

Coser, L. (1956). The functions of social conflict. New York: The Free Press.

Dovidio, J. F., Johnson, J. D., Gaertner, S. L., Pearson, A. R., Saguy, T., \& AshburnNardo, L. (2010). Empathy and intergroup relations. In M. Mikulincer \& P. R. Shaver (Eds.), Prosocial motives, emotions, and behavior: The better angels of our nature (pp. 393-408). Washington, DC: American Psychological Association, doi: 10.1037/12061-020.

Dziobek, I., Rogers, K., Fleck, S., Bahnemann, M., Heekeren, H. R., Wolf, O. T., \& Convit, A. (2008). Dissociation of cognitive and emotional empathy in adults with Asperger syndrome using the Multifaceted Empathy Test (MET). Journal of Autism and Developmental Disorders, 38(3), 464-473, doi: 10.1007/ s10803-007-0486-x.

Finlay, K. A, \& Stephan, W. G. (2000). Improving intergroup relations: The effects of empathy on racial attitudes. Journal of Applied Social Psychology, 30, 17201737, doi: 10.1111/j.1559-1816.2000.tb02464.x.

Fiske, S. T., Cuddy, A. J. C., Glick, P., \& Xu, J. (2002). A model of (often mixed) stereotype content: Competence and warmth respectively follow from perceived status and competition. Journal of Personality and Social Psychology, 82(6), 878-902, doi: 10.1037//0022-3514.82.6.878.

Giovannini, D., Pintus, A., \& Rinaldi, L. (2007). Immigrazione e pregiudizio: l'atteggiamento dei datori di lavoro verso i dipendenti extracomunitari in piccole e medie imprese trentine. In R. Brown, D. Capozza, \& O. Licciardello (a cura di), Immigrazione, acculturazione, modalità di contatto (pp. 233-248). Milano: FrancoAngeli.

Hughes, M. A., \& Garrett, D. E. (1990). Intercoder reliability estimation approaches in marketing: A genealizability theory framework for quantitative data. Journal of Marketing Research, 27(2), 185-195, doi: 10.2307/3172845.

Intraversato, A., \& Lucisano, P. (2013). Gli anni di Eco. Riflessioni sull'uso di prove strutturate con risposte chiuse e aperte a margine di una ricerca sulla comprensione della lettura. Journal of Educational, Cultural and Psychological Studies, 7, 23-43, doi: 10.7358/ecps-2013-007-intr.

INVALSI (2012a). OCSE PISA 2012. Rapporto nazionale. Retrieved from: http:// www.invalsi.it/invalsi/ri/pisa2012/rappnaz/Rapporto_NAZIONALE_ OCSE_PISA2012.pdf 
INVALSI (2012b). Indagini IEA 2011 PIRLS e TIMSS: i risultati degli studenti italiani in lettura, matematica e scienze. Retrieved from: http://www.invalsi.it/ invalsi/ri/timss2011/documenti/Rapporto_PIRLS_TIMSS.pdf

INVALSI (2015). Rilevazioni nazionali degli apprendimenti 2014-2015. Retrieved from: https://invalsi-areaprove.cineca.it/docs/attach/035_Rapporto_Prove_ INVALSI_2015.pdf

Isac, M. M., Maslowski, R., \& van der Werf, G. (2012). Native students attitudes towards equal rights for immigrants. A study in 18 European countries. JSSE Journal of Social Science Education, 11(1), 7-22, doi: 10.2390/jsse-v11-i1-1189.

Johnson-Laird, P. N. (1989). Mental models. In M. Posner (Ed.), Foundations of cognitive science (pp. 469-499). Cambridge, MA: MIT Press, doi: 10.1002/15206696(199107)27:3.

Kövecses, Z. (2010). Metaphor: A practical introduction (2nd ed.). New York: Oxford University Press, doi: 10.1017/S0047404503254051.

Krippendorff, K. (1983). Analisi del contenuto: Introduzione metodologica. Torino: ERI.

Krosnick, J. A., Judd, C. M., \& Wittenbrink, B. (2005). The measurement of attitudes. In D. Albarracin, B. T. Johnson, \& M. P. Zanna (Eds.), The handbook of attitudes (pp. 21-76). Mahwah, NJ: Erlbaum, doi: 10.4324/9781410612823.

Lakoff, G. (1993). The contemporary theory of metaphor. Metaphor and thought. In A. Ortony (Ed.), Metaphor and thought (2nd ed., pp. 202-251). Cambridge: Cambridge University Press, doi: http://dx.doi.org/10.1017/ CBO9781139173865.

Lakoff, G., \& Johnson, M. (1980). Metaphors we live by. Chicago: University of Chicago Press, doi: 10.1525/aa.1982.84.4.02a00600.

La Marca, A. (2014). Nuovo realismo e metodi di ricerca mista. Journal of Educational, Cultural and Psychological Studies, 9, 397-416, doi: 10.7358/ecps2014-009-lama.

Lebart, L., Piron, M., \& Morineau, A. (1995). Statistique exploratoire multidimensionelle. Paris: Dunod.

Lee, T. L., \& Fiske, S. T. (2006). Not an outgroup, not yet an ingroup: Immigrants in the Stereotype Content Model. International Journal of Intercultural Relations, 30, 751-768, doi: 10.1016/j.ijintrel.2006.06.005.

Lucidi, F., Alivernini, F., \& Pedon, A. (2008). Metodologia della ricerca qualitativa. Roma: il Mulino.

Lucisano, P., \& Salerni, A. (2003). Metodologia della ricerca in educazione e formazione. Roma: Carocci.

Mayring, P. (2000). Qualitative Content Analysis. Forum Qualitative Sozialforschung / Forum: Qualitative Social Research, 1(2), Art. 20. Retrieved from: http://nbn-resolving.de/urn:nbn:de:0114-fqs0002204 
Mezzadra, S. (2006). Diritto di fuga. Migrazioni, cittadinanza, globalizzazione. Verona: Ombre corte.

MIUR - Fondazione ISMU (2015). Alunni con cittadinanza non italiana - Tra difficoltà e successi. Rapporto nazionale a.s. 2013/2014 (Quaderni ISMU, 1). Retrieved from: http://www.istruzione.it/allegati/2015/Rapporto_alunni_ cittadinanza_non_italiana_2013_14.pdf

Moscovici, S. (1984). The phenomenon of social representations. In R. Farr \& S. Moscovici (Eds.), Social representations (pp. 3-70). Cambridge: Cambridge University Press.

Moscovici, S. (1988). Notes towards a description of social representations. European Journal of Social Psychology, 18, 211-250, doi: 10.1002/ejsp.2420180303.

Mosso, C. (2007). Immigrati: minaccia o risorsa per il sistema sociale? In R. Brown, D. Capozza, \& O. Licciardello (a cura di), Immigrazione, acculturazione, modalità di contatto (pp. 266-279). Milano: FrancoAngeli.

Neuendorf, K. A. (2002). The content analysis guidebook. Thousand Oaks, CA: Sage.

Pedersen, A., Beven, J. P., Walker, I., \& Griffiths, B. (2004). Attitudes toward indigenous Australians: The role of empathy and guilt. Journal of Community \& Applied Social Psychology, 14(4), 233-249, doi: 10.1002/casp.771.

Pettigrew, T. F. (1998). Intergroup contact theory. Annual Review of Psychology, 49, 65-85, doi: 10.1146/annurev.psych.49.1.65.

Pettigrew, T. F., \& Tropp, L. R. (2006). A meta-analytic test of intergroup contact theory. Journal of Personality and Social Psychology, 90(5), 751-783, doi: 10.1037/0022-3514.90.5.751.

Portes, A., Fernandez-Kelly, P., \& Haller, W. (2005). Segmented assimilation on the ground: The new second generation in early adulthood. Ethnic and Racial Studies, 28(6), 1000-1040, doi: 10.1080/01419870500224117.

Portes, A., \& Zhou, M. (1993). The new second generation: Segmented assimilation and its variants. The Annals of the American Academy of Political and Social Science, 530(1), 74-96, doi: 10.1177/0002716293530001006.

Quillan, L. (1995). Prejudice as a response to perceived group threat: Population composition and anti-immigrant and racial prejudice in Europe. American Sociological Review, 60, 586-611, doi: 10.2307/2096296.

Scalera, V., Palmerio, L., \& Alivernini, F. (2007). La mia scuola è un’orchestra. Studio di validazione del questionario «Immagini di scuola attraverso le metafore»: ulteriori approfondimenti. Rivista di Psicologia dell'Educazione e della Formazione, 9(2), 5-36, doi: 10.4399/97888548159881.

Schulz, W., Ainley, J., \& Fraillon, J. (Eds.). (2011). ICCS 2009 Technical report. Amsterdam: International Association for the Evaluation of Educational Achievement. Retrieved from: http://www.iea.nl/fileadmin/user_upload/ Publications/Electronic_versions/ICCS_2009_Technical_Report.pdf 
Schwartz, S. J., \& Zamboanga, B. L. (2008). Testing Berry's model of acculturation: A confirmatory latent class approach. Cultural Diversity and Ethnic Minority Psychology, 14(4), 275-285, doi: 10.1037/a0012818.

Sherif, M. (1966). Group conflict and cooperation. Their social psychology. London: Routledge \& K. Paul.

Sherif, M., \& Sherif, C. W. (1969). Social psychology. New York: Harper \& Row.

Stephan, W. G., \& Stephan, C. W. (2000). An integrated threat theory of prejudice. In S. Oskamp (Ed.), Reducing prejudice and discrimination (pp. 23-45). Mahwah, NJ: Lawrence Erlbaum Associates, doi: 10.1177/0022022100031002006.

Stephan, W. G., \& Stephan, C. W. (2001). Improving intergroup relations. Thousand Oaks, CA: Sage.

Stephan, W. G., Ybarra, O., \& Bachman, G. (1999). Prejudice toward immigrants: An integrated threat theory. Journal of Applied Social Psychology, 29, 559-576, doi: http://dx.doi.org/10.1111/j.1559-1816.1999.tb00107.x.

Tajfel, H. (1982). Social psychology of intergroup relations. Annual Review of Psychology, 33(1), 1-39, doi: 10.1146/annurev.ps.33.020182.000245.

Tajfel, H., \& Turner, J. C. (1979). An integrative theory of intergroup conflict. In W. G. Austin \& S. Worchel (Eds.), The social psychology of intergroup relations (pp. 33-47). Monterey, CA: Brooks-Cole.

Voci, A., \& Hewstone, M. (2007). L'importanza dell'empatia nella relazione tra contatto e riduzione del pregiudizio. In R. Brown, D. Capozza, \& O. Licciardello (a cura di), Immigrazione, acculturazione, modalità di contatto (pp. 33-49). Milano: FrancoAngeli.

Vosniadou, S., \& Ortony, A. (1989). Similarity and analogical reasoning. New York: Cambridge University Press, doi: http://dx.doi.org/10.1017/ CBO9780511529863.

Weber, R. P. (1990). Basic content analysis (2nd ed.). Newbury Park, CA: Sage, doi: http://dx.doi.org/10.4135/9781412983488.n4.

\section{RiassunTO}

L'obiettivo di questo studio è quello di identificare le rappresentazioni di giovani studenti italiani rispetto agli immigrati e di valutare la relazione di queste immagini con il background familiare di provenienza degli alunni. I dati si basano su un campione di studenti italiani $(N=841)$. Agli studenti è stata posta una domanda aperta che chiedeva di completare una similitudine relativa agli immigrati. La metodologia utilizzata è stata di tipo misto e ha previsto: (i) un'analisi del contenuto qualitativa; (ii) un'analisi quantitativa, che ha indagato la relazione tra le categorie individuate attraverso l'analisi del contenuto e lo status socioeconomico dello studente. I risultati mostrano un insieme di 
rappresentazioni molto vario e articolato. I contenuti delle metafore prodotte dagli studenti spaziano da tematiche riconducibili ad una contrapposizione "noi-loro»; a immagini centrate su dimensioni di tolleranza e uguaglianza; ad aree semantiche che prevedono invece una componente di tipo emotivo. Il background socioeconomico dello studente ha mostrato un pattern di relazione specifico per alcune similitudini. La metodologia di ricerca mista adottata è risultata essere efficace rispetto agli obiettivi dello studio, combinando la ricchezza qualitativa delle risposte a una domanda aperta basata su una metafora con strumenti statistici affidabili.

Parole chiave: Immigrati, Metafore, Metodi misti, Rappresentazioni mentali, Status socioeconomico.

How to cite this Paper: Cavicchiolo, E., Alivernini, F., \& Manganelli, S. (2016). Immigrants are like ... The representation of immigrants in Italy: The metaphors used by students and their family backgrounds [Gli immigrati sono come ... La rappresentazione degli immigrati in Italia: le metafore degli studenti e il background familiare di provenienza]. Journal of Educational, Cultural and Psychological Studies, 13, 163-190. doi: 10.7358/ecps-2016-013-cavi 